\title{
A Principled Approach to Score Level Fusion in Multimodal Biometric Systems
}

\author{
Sarat C. Dass ${ }^{1}$, Karthik Nandakumar ${ }^{2}$, and Anil K. Jain ${ }^{2}$ \\ 1 Department of Statistics and Probability \\ sdass@stt.msu.edu \\ 2 Department of Computer Science and Engineering \\ \{nandakum, jain\}@cse.msu.edu \\ Michigan State University, MI - 48824, U.S.A.
}

\begin{abstract}
A multimodal biometric system integrates information from multiple biometric sources to compensate for the limitations in performance of each individual biometric system. We propose an optimal framework for combining the matching scores from multiple modalities using the likelihood ratio statistic computed using the generalized densities estimated from the genuine and impostor matching scores. The motivation for using generalized densities is that some parts of the score distributions can be discrete in nature; thus, estimating the distribution using continuous densities may be inappropriate. We present two approaches for combining evidence based on generalized densities: (i) the product rule, which assumes independence between the individual modalities, and (ii) copula models, which consider the dependence between the matching scores of multiple modalities. Experiments on the MSU and NIST multimodal databases show that both fusion rules achieve consistently high performance without adjusting for optimal weights for fusion and score normalization on a case-by-case basis.
\end{abstract}

Keywords: Biometric recognition, multimodal biometric systems, fusion, Gaussian copula models, Generalized densities, Neyman-Pearson theorem.

\section{Introduction}

Biometrics refers to the automatic identification of an individual based on his/her physiological traits [1]. Biometric systems based on a single source of information (unimodal systems) suffer from limitations like the lack of uniqueness, non-universality and noisy data [2] and hence, may not be able to achieve the desired performance requirements of real-world applications. In contrast, multimodal biometric systems combine information from its component modalities to arrive at a decision [3]. Several studies [4-8] have demonstrated that by consolidating information from multiple sources, better performance can be achieved compared to the individual unimodal systems. In a multimodal biometric system, integration can be done at (i) feature level, (ii) matching score level, or (iii) 
decision level. Matching score level fusion is commonly preferred because matching scores are easily available and contain sufficient information to distinguish between a genuine and an impostor case. Given a number of biometric systems, one can generate matching scores for a pre-specified number of users even without knowing the underlying feature extraction and matching algorithms of each biometric system. Thus, combining information contained in the matching scores seems both feasible and practical.

We propose a framework for optimally combining the matching scores from multiple modalities based on generalized densities estimated from the genuine and impostor matching scores. The motivation for using generalized densities is that some parts of the score distributions can be discrete in nature. As a result, estimating the densities using continuous density functions can be inappropriate. We present two approaches for combining evidence based on generalized densities: (i) the product rule, which assumes independence between the individual modalities, and (ii) copula models, which parametrically model the dependence between the matching scores of multiple modalities. Our proposed method bypasses the need for score normalization and selection of optimal weights for the score combination on a case-by-case basis $[3,9,10]$, and therefore, is a more principled approach with performance comparable to the commonly used fusion methods. Experiments have shown that our method achieves consistently high performance over the MSU and NIST multimodal databases.

\section{Generalized Densities}

\subsection{Estimation of Marginal Distributions}

Let $X$ be a generic matching score with distribution function $F$, i.e., $P(X \leq x)=$ $F(x)$. We denote the genuine (impostor) matching score by $X_{g e n}\left(X_{i m p}\right)$ and the corresponding distribution function by $F_{g e n}\left(F_{i m p}\right)$. Assuming that $F_{g e n}(x)$ and $F_{i m p}(x)$ have densities $f_{g e n}(x)$ and $f_{i m p}(x)$, respectively, the Neyman-Pearson theorem states that the optimal ROC curve is the one corresponding to the likelihood ratio statistic $N P(x)=f_{\text {gen }}(x) / f_{\text {imp }}(x)$ [11]. The ROC curve corresponding to $N P(x)$ has the highest genuine accept rate (GAR) for every given value of the false accept rate (FAR) compared to any other statistic $U(x) \neq N P(x)$ (this is true even for the original matching scores corresponding to $U(x)=x$ ).

However, when $f_{g e n}(x)$ and $f_{i m p}(x)$ are unknown (which is typically the case) and are estimated from the observed matching scores, the ROC corresponding to $N P(x)$ may turn out to be suboptimal. This is mainly due to the large errors in the estimation of $f_{g e n}(x)$ and $f_{i m p}(x)$. Thus, for a set of genuine and impostor matching scores, it is important to be able to estimate $f_{g e n}(x)$ and $f_{\text {imp }}(x)$ reliably and accurately. Previous studies by Griffin [11] and Prabhakar et al. [12] assume that the distribution function $F$ has a continuous density with no discrete components. In reality, most matching algorithms apply thresholds at various stages in the matching process. When the required threshold conditions are not met, specific matching scores are output by the matcher (e.g., some fingerprint matchers produce a score of zero if the number of extracted minutiae 


\section{To appear in Proceedings of AVBPA 2005}

is less than a threshold). This leads to discrete components in the matching score distribution that cannot be modeled accurately using a continuous density function. A score value $x_{0}$ is said to be discrete if $P\left(X=x_{0}\right)=p>0$. It is easy to see that $F$ cannot be represented by a density function in a neighborhood of $x_{0}$ (since this would imply that $P\left(X=x_{0}\right)=0$ ). Thus, discrete components need to be detected and modeled separately to avoid large errors in estimating $f_{g e n}(x)$ and $f_{i m p}(x)$. Our approach consists of detecting discrete components in the genuine and impostor matching score distributions, and then modeling the observed distribution of matching scores as a mixture of discrete and continuous components. Hence, this approach generalizes the work of $[11,12]$.

The following methodology can model a distribution based on a generic set of observed scores. For a fixed threshold $T$, the discrete values are identified as those values $x_{0}$ with $P\left(X=x_{0}\right)>T$, where $0 \leq T \leq 1$. Since the underlying matching score distribution is unknown, we estimate the probability $P\left(X=x_{0}\right)$ by $\frac{N\left(x_{0}\right)}{N}$, where $N\left(x_{0}\right)$ is the number of observations in the data set that equals $x_{0}$, and $N$ is the total number of observations. The collection of all discrete components for a matching score distribution will be denoted by

$$
\mathcal{D} \equiv\left\{x_{0}: \frac{N\left(x_{0}\right)}{N}>T\right\}
$$

The discrete components constitute a proportion $p_{D} \equiv \sum_{x_{0} \in \mathcal{D}} \frac{N\left(x_{0}\right)}{N}$ of the total observations. We obtain the collection $\mathcal{C}$ by removing all discrete components from the entire data set. The scores in $\mathcal{C}$ constitute a proportion $p_{C} \equiv 1-p_{D}$ of the entire data set, and they are used to estimate the continuous component of the distribution $\left(F_{C}(x)\right)$ and the corresponding density $\left(f_{c}(x)\right)$. A non-parametric kernel density estimate of $f_{c}(x)$ is obtained from $\mathcal{C}$ as follows. The empirical distribution function for the observations in $\mathcal{C}$ is computed as

$$
\hat{F}_{C}(x)=\frac{1}{N_{C}} \sum_{s \in \mathcal{C}} I\{s \leq x\},
$$

where $I\{s \leq x\}=1$ if $s \leq x$, and $=0$, otherwise; also, $N_{C} \equiv N p_{C}$. Note that $\hat{F}_{C}(x)=0 \forall x<s_{\min }$ and $\hat{F}_{C}(x)=1 \forall x \geq s_{\max }$, where $s_{\min }$ and $s_{\max }$, respectively, are the minimum and maximum of the observations in $\mathcal{C}$. For values of $x, s_{\min }<x<s_{\max }$, not contained in $\mathcal{C}, \hat{F}_{C}(x)$, is obtained by linear interpolation. Next, $B$ samples are simulated from $\hat{F}_{C}(x)$, and the density estimate of $f_{C}(x), \hat{f}_{C}(x)$, is obtained from the simulated samples using a Gaussian kernel density estimator. The optimal bandwidth, $h$, is obtained using the "solve-theequation" bandwidth estimator [13], which is an automatic bandwidth selector that prevents oversmoothing and preserves important features of the distribution of matching scores (see Figure 1). The generalized density is defined as

$$
l(x)=p_{C} \hat{f}_{C}(x)+\sum_{x_{0} \in \mathcal{D}} \frac{N\left(x_{0}\right)}{N} \cdot I\left\{x=x_{0}\right\},
$$




\section{To appear in Proceedings of AVBPA 2005}

where $I\left\{x=x_{0}\right\}=1$ if $x=x_{0}$, and $=0$, otherwise. The distribution function corresponding to the generalized density is defined as

$$
L(x)=p_{C} \int_{-\infty}^{x} \hat{f}_{C}(u) d u+\sum_{x_{0} \in \mathcal{D}, x_{0} \leq x} \frac{N\left(x_{0}\right)}{N} .
$$

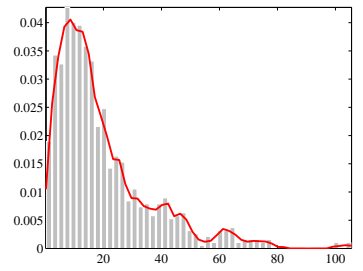

(a)

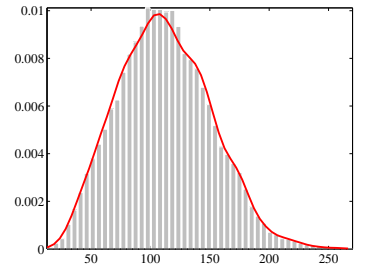

(d)

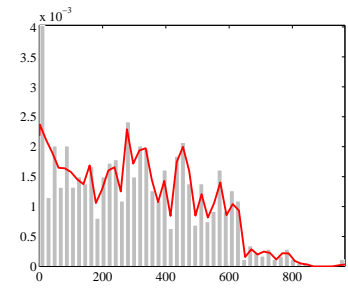

(b)

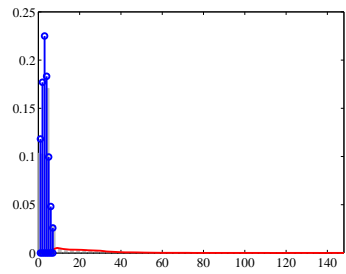

(e)

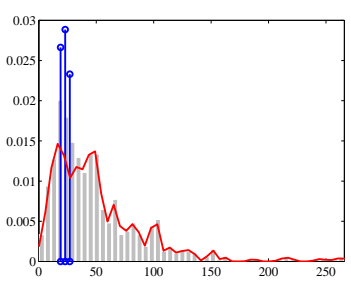

(c)

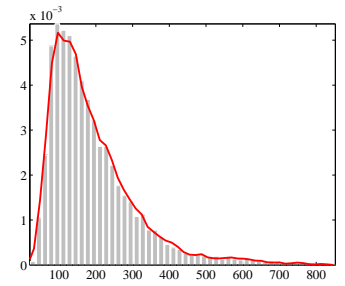

(f)

Fig. 1. Histograms of matching scores and corresponding generalized density estimates. Row 1: Histograms of genuine scores for face (a), finger (b), and hand-geometry (c). Row 2: Histograms of impostor scores for face (d), finger (e), and hand-geometry (f). The solid line is the estimated density using the kernel density estimator, and the spikes in (c) and (e) correspond to detected discrete components. Note that no preprocessing of the matching score data (including the conversion of distance measures into similarity scores) was performed before density estimation.

For a multimodal system with $K$ modalities, the generalized densities and distributions estimated for the genuine (impostor) scores for the $k^{\text {th }}$ modality will be denoted by $l_{g e n, k}(x)$ and $L_{g e n, k}(x)\left(l_{i m p, k}(x)\right.$ and $\left.L_{i m p, k}(x)\right)$, respectively, for $k=1,2, \ldots, K$. Figures 1 (a)-(f) give the plots of $l_{g e n, k}(x)$ and $l_{i m p, k}(x)$ for the distribution of observed genuine and impostor matching scores for $K=3$ modalities of the MSU-Multimodal database (see Section 4). Figures 1 (a)-(f) also give the histograms of the genuine and impostor matching scores for the three modalities. The "spikes" (see Figure 1 (c) and (e)) represent the detected discrete components and have a height greater than the threshold $T=0.02$. Note that the individual "spikes" cannot be represented by a continuous density function. Forcing a continuous density estimate for these values will result in gross estimation errors and yield suboptimal ROC curves. 


\subsection{Joint Density Estimation using Copula Models}

The methodology described in Section 2.1 only estimates the marginal score distributions of each of the $K$ modalities without estimating the joint distribution. One way to estimate the joint distribution of matching scores is by using copula models [14]. Let $H_{1}, H_{2}, \ldots, H_{K}$ be $K$ continuous distribution functions on the real line and $H$ be a $K$-dimensional distribution function with the $k^{\text {th }}$ marginal given by $H_{k}$ for $k=1,2, \ldots, K$. According to Sklar's Theorem [14], there exists a unique function $C\left(u_{1}, u_{2}, \ldots, u_{K}\right)$ from $[0,1]^{K}$ to $[0,1]$ satisfying

$$
H\left(s_{1}, s_{2}, \ldots, s_{K}\right)=C\left(H_{1}\left(s_{1}\right), H_{2}\left(s_{2}\right), \ldots, H_{K}\left(s_{K}\right)\right),
$$

where $s_{1}, s_{2}, \ldots, s_{K}$ are $K$ real numbers. The function $C$ is known as a $K$-copula function that "couples" the one-dimensional distributions functions $H_{1}, H_{2}, \ldots$, $H_{K}$ to obtain the $K$-variate function $H$. Equation (5) can also be used to construct $K$-dimensional distribution functions $H$ whose marginals are the distributions $H_{1}, H_{2}, \ldots, H_{K}$ : choose a copula function $C$ and define $H$ as in (5).

Copula functions are effective in modeling the joint distribution when the marginal distributions are non-normal and do not have a parametric form (as is usually the case for biometric data, see Figure 1). The family of copulas considered in this paper is the $K$-dimensional multivariate Gaussian copulas [15]. These functions can represent a variety of dependence structures using a $K \times K$ correlation matrix $R$. The $K$-dimensional Gaussian copula function with correlation matrix $R$ is given by

$$
C_{R}^{K}\left(u_{1}, u_{2}, \ldots, u_{K}\right)=\Phi_{R}^{K}\left(\Phi^{-1}\left(u_{1}\right), \Phi^{-1}\left(u_{2}\right), \ldots, \Phi^{-1}\left(u_{K}\right)\right)
$$

where each $u_{k} \in[0,1]$ for $k=1,2, \ldots, K, \Phi(\cdot)$ is the distribution function of the standard normal, $\Phi^{-1}(\cdot)$ is its inverse, and $\Phi_{R}^{K}$ is the $K$-dimensional distribution function of a random vector $\mathcal{Z}=\left(Z_{1}, Z_{2}, \ldots, Z_{K}\right)^{T}$ with component means and variances given by 0 and 1 , respectively. The $(m, n)$-th entry of $R, \rho_{m n}$, measures the degree of correlation between the $m$-th and $n$-th components for $m, n=1,2, \ldots, K$. In practice, $\rho_{m n}$ will be unknown and hence, will be estimated using the product moment correlation of normal quantiles corresponding to the observed scores from the $K$ modalities.

We denote the density of $C_{R}^{K}$ by

$$
\begin{aligned}
c_{R}^{K}\left(u_{1}, u_{2}, \ldots, u_{K}\right) & \equiv \frac{\partial C_{R}^{K}\left(u_{1}, u_{2}, \ldots, u_{K}\right)}{\partial u_{1} \partial u_{2} \ldots \partial u_{K}} \\
& =\frac{\phi_{R}^{K}\left(\Phi^{-1}\left(u_{1}\right), \Phi^{-1}\left(u_{2}\right), \ldots, \Phi^{-1}\left(u_{K}\right)\right)}{\prod_{k=1}^{K} \phi\left(\Phi^{-1}\left(u_{k}\right)\right)},
\end{aligned}
$$

where $\phi_{R}^{K}\left(x_{1}, x_{2}, \ldots, x_{K}\right)$ is the joint probability density function of the $K$ variate normal distribution with mean 0 and covariance matrix $R$, and $\phi(x)$ is the standard normal density function. We will assume that the joint distribution function of genuine (impostor) matching scores for $K$ modalities, $F_{g e n}^{K}\left(F_{i m p}^{K}\right)$, is of the form (5) for some correlation matrix $R_{0}\left(R_{1}\right)$. For the genuine (impostor) case, $H_{k}$ will be estimated by $L_{g e n, k}(x)\left(L_{i m p, k}(x)\right)$ for $k=1,2, \ldots, K$. 


\section{Fusion Based On Generalized Densities}

Two methods of fusion have been considered in this paper. The first method assumes independence between the $K$ biometric modalities and combines the estimated marginal densities using the product rule. For the matching score set $\mathcal{S}=\left(S_{1}, S_{2}, \ldots, S_{K}\right)$, the product fusion score of $\mathcal{S}, P F S(\mathcal{S})$, is given by

$$
\operatorname{PFS}(\mathcal{S})=\prod_{k=1}^{K} \frac{l_{g e n, k}\left(S_{k}\right)}{l_{i m p, k}\left(S_{k}\right)}
$$

where $l_{g e n, k}(\cdot)$ and $l_{i m p, k}(\cdot)$ are the estimates of generalized densities of the genuine and impostor scores of the $k^{t h}$ biometric modality.

The copula fusion rule combines the individual modalities using the estimated Gaussian copula functions for the score distributions. The copula fusion score of a matching score set $\mathcal{S}=\left(S_{1}, S_{2}, \ldots, S_{K}\right), C F S(\mathcal{S})$, is given by $C F S(\mathcal{S})=$

$$
\operatorname{PFS}(\mathcal{S}) \cdot \frac{c_{R_{0}}^{K}\left(\Phi^{-1}\left(L_{g e n, 1}\left(S_{1}\right)\right), \Phi^{-1}\left(L_{g e n, 2}\left(S_{2}\right)\right), \ldots, \Phi^{-1}\left(L_{g e n, K}\left(S_{K}\right)\right)\right)}{c_{R_{1}}^{K}\left(\Phi^{-1}\left(L_{i m p, 1}\left(S_{1}\right)\right), \Phi^{-1}\left(L_{i m p, 2}\left(S_{2}\right)\right), \ldots, \Phi^{-1}\left(L_{i m p, K}\left(S_{K}\right)\right)\right)},
$$

where $L_{g e n, k}\left(S_{k}\right)$ and $L_{i m p, k}\left(S_{k}\right)$ are, respectively, the estimates of generalized distribution functions for the $k^{t h}$ biometric modality, and $c_{R}^{K}$ is the density of $C_{R}^{K}$ as defined in (7). This fusion rule assumes that the Gaussian copula functions can adequately model the dependence between the $K$ biometric modalities.

\section{Experimental Results}

Experiments on fusion of matching scores using rules (8) and (9) were carried out on two different multimodal databases. For each experiment, $70 \%$ of the genuine and impostor matching scores were randomly selected to be the training set for the estimation of the generalized densities and the correlation matrices. The remaining $30 \%$ of the genuine and impostor scores were used to generate the ROC curves. This training-testing partition was repeated 20 times and the performance results reported for each value of FAR are the median GAR values.

\subsection{Databases}

Table 1 summarizes the multimodal databases used in our experiments. The first database (referred to as the MSU-Multimodal database) consisted of 100 "virtual" subjects each providing five samples of face, fingerprint (left-index) and hand-geometry modalities. Face images were represented as eigenfaces [16] and the Euclidean distance between the eigen coefficients of the template-query pair was used as the distance metric. Minutia points were extracted from fingerprint images and the elastic string matching technique [17] was used for computing the similarity between two minutia point patterns. Fourteen features describing the geometry of the hand shape [18] were extracted from the hand images and Euclidean distance was computed for each template-query pair. 
Table 1. Summary of Multimodal Databases Used

\begin{tabular}{|l|c|c|c|}
\hline \multicolumn{1}{|c|}{ Database } & Modalities & K & No. of Users \\
\hline MSU-Multimodal & Fingerprint, Face, Hand-geometry & 3 & 100 \\
\hline NIST-Multimodal & $\begin{array}{c}\text { Fingerprint (Two fingers), } \\
\text { Face (Two matchers) }\end{array}$ & 4 & 517 \\
\hline
\end{tabular}

Experiments were also conducted on the first partition of the Biometric Scores Set - Release I (BSSR1) released by NIST [19]. The NIST-Multimodal database consists of 517 users and is "truly multimodal" in the sense that the fingerprint and face images used for genuine matching score computation came from the same individual. One fingerprint score was obtained by comparing a pair of impressions of the left index finger and another score was obtained by comparing impressions of the right index finger. Two different face matchers were applied to compute the similarity between frontal face images. Even though the number of subjects in the NIST database is relatively large, there are only two samples per subject. So the number of genuine scores is still rather small.

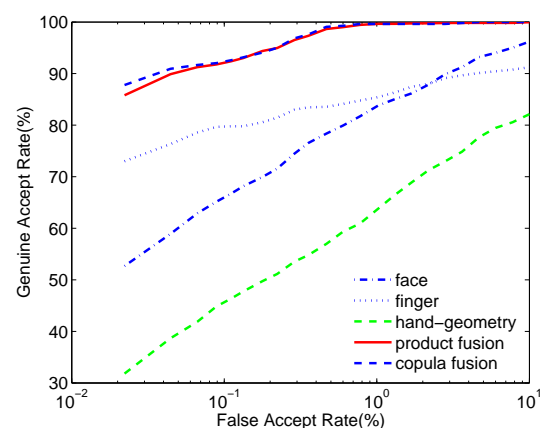

(a)

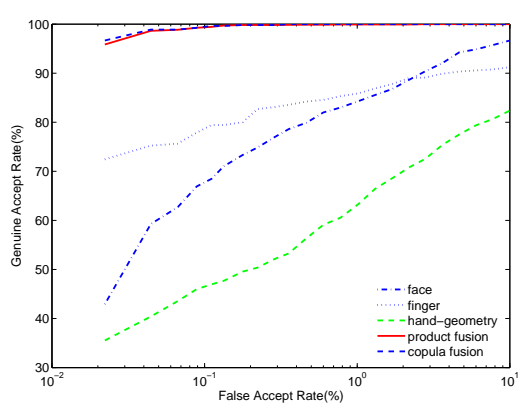

(b)

Fig. 2. Performance of product and copula fusion on the MSU-Multimodal database based on (a) continuous and (b) generalized density estimates.

Figure 2 gives the ROC curves for the two fusion rules and the ROC curves based on the matching scores of individual modalities for the MSU-Multimodal database. Figure 2(a) shows the recognition performance when the genuine and impostor score distributions of the three modalities are modeled purely by continuous densities. The performance improvement obtained by modeling the matching score distributions as a mixture of discrete and continuous components (generalized densities) can be observed by comparing Figures 2(a) and 2(b). The ROC curves for the two fusion rules on the NIST-Multimodal database are shown in Figure $3(a)$. We see that both fusion rules give significantly better matching performance compared to the best single modality in each database. We also observe that the best single modality in both the databases is uncorrelated to the other modalities. For the MSU-Multimodal database, the estimates of the correlation of the best single modality (fingerprint) with the other two modalities (face and hand-geometry) are -0.01 and -0.11 for the genuine scores, and 


\section{To appear in Proceedings of AVBPA 2005}

-0.05 and -0.04 for the impostor scores. For the NIST-Multimodal database (the best single modality is finger 2), the correlation estimates (with face1, face2, and finger 1 modalities, respectively) are $-0.02,-0.06$, and 0.43 for the genuine cases and $0.04,0.02$, and 0.14 for the impostor cases. Since the fusion is driven mostly by the best modality, the fact that this modality is approximately independent of the others means that the product and copula fusion rules should be comparable to each other as reflected by the ROC curves in Figures 2 and 3(a).

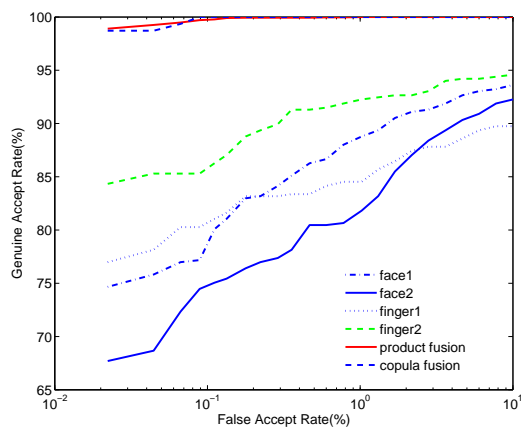

(a)

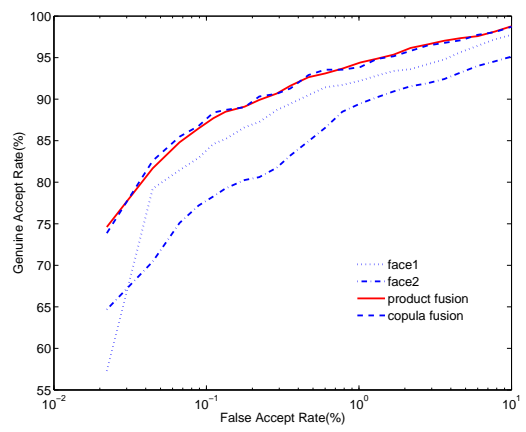

(b)

Fig. 3. ROC curves for the NIST-Multimodal database; (a) all four modalities (b) only face 1 and face 2 modalities.

In order to study the usefulness of the copula fusion rule, we analyzed the fusion results of the face 1 and face 2 modalities of the NIST-Multimodal database (see Figure $3(b)$ ). This pair had the highest degree of correlation among all pairs in the two databases $(0.75$ and 0.29 for the genuine and impostor scores, respectively). We observed that even in this case, the performance difference between the product and copula fusion rules is not significant. This may be due to the fact that although incorporating the correlation between the multiple matching scores into the fusion rule should result in better performance than fusion based on the independence assumption, the difference will be significant only in a few cases. The following simulations illustrate this fact. Let the matching scores of two biometric modalities follow the bivariate normal distribution with the following parameters (these values were chosen so as to closely model the matching scores of face 1 and face 2 modalities in the NIST-Multimodal database):

$$
\begin{gathered}
S_{g e n} \sim N\left(\mu_{\text {gen }}=\left[\begin{array}{c}
0.72 \\
76.78
\end{array}\right], \Sigma_{g e n}=\left[\begin{array}{cc}
0.006 & 0.15 \\
0.15 & 8.31
\end{array}\right]\right), \\
S_{i m p} \sim N\left(\mu_{i m p}=\left[\begin{array}{c}
0.53 \\
66.87
\end{array}\right], \Sigma_{i m p}=\left[\begin{array}{cc}
0.0015 & 0.03 \\
0.03 & 9.45
\end{array}\right]\right) .
\end{gathered}
$$

We generated 100, 000 genuine and 100,000 impostor scores from the above distributions. In the first experiment, we assume that the parameters in equations (10) and (11) are known. The likelihood ratios were computed by utilizing the full $\Sigma$, and under the independence assumption (non-diagonal elements of 
$\Sigma$ matrix are set to zero). The ROC curves for these two cases are plotted in Figure 4(a) which show that for this specific parameter set, utilizing the correlation information does not substantially improve the performance. On the other hand, if the $\Sigma_{\text {gen }}$ matrix is changed to $\left[\begin{array}{cc}0.006 & 0.20 \\ 0.20 & 8.31\end{array}\right]$ (corresponds to increasing the correlation between the genuine matching scores of the two modalities $\left(\rho_{\text {gen }}\right)$ from 0.75 to 0.90 ), we observe that fusion accounting for the correlation provides substantial improvement over the independence case (see Figure $4(b)$ ). Now, if we estimate the parameters in equations (10) and (11) using the simulated data, the copula fusion rule outperforms the product rule as shown in Figure $4(c)$. These experiments illustrate that improvement in the recognition performance by using copula fusion rule depends on the underlying distribution of the matching scores. In the general case, the copula rule will perform at least as good as the product rule, provided there is sufficient amount of training data to estimate the correlation matrices accurately.

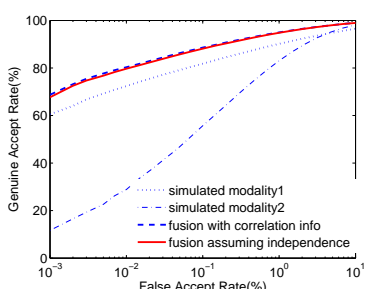

(a)

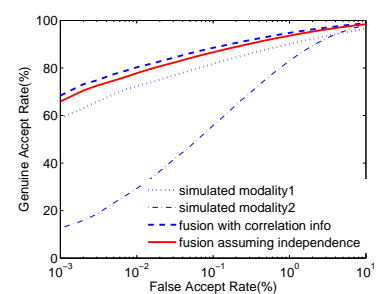

(b)

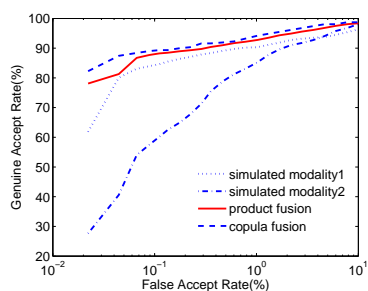

(c)

Fig. 4. ROC curves for the simulated data; (a) fusion with true parameters when $\rho_{\text {gen }}=0.75$, (b) fusion with true parameters when $\rho_{\text {gen }}=0.90$ and (c) fusion using estimated parameters when $\rho_{\text {gen }}=0.90$.

\section{Summary}

Based on the generalized density estimates of the genuine and impostor matching scores, two methods of fusion that follow the Neyman-Pearson rule are described. The first fusion rule computes the product of the likelihood ratios for each component modality of a multimodal system and is optimal when the modalities are independent of each other. The second fusion rule assumes that the generalized joint density of matching scores can be modeled using a Gaussian copula function and is a generalization of the product rule when the component modalities are not independent. Experimental results indicate that the two fusion rules achieve better performance compared to the single best modality in both the databases. The proposed method bypasses the need to perform score normalization and choosing optimal combination weights for each modality on a case-by-case basis. In this sense, the proposed solution is a principled and general approach that is optimal when the genuine and impostor matching score distributions are either known or can be estimated with high accuracy. 


\section{To appear in Proceedings of AVBPA 2005}

\section{Acknowledgments}

This research is partially supported by the NSF ITR grant 0312646 and the Center for Identification Technology Research (CITeR).

\section{References}

1. Jain, A.K., Ross, A., Prabhakar, S.: An Introduction to Biometric Recognition. IEEE Transactions on Circuits and Systems for Video Technology, Special Issue on Image- and Video-Based Biometrics 14 (2004) 4-20

2. Jain, A.K., Ross, A.: Multibiometric Systems. Communications of the ACM, Special Issue on Multimodal Interfaces 47 (2004) 34-40

3. Ross, A., Jain, A.K.: Information Fusion in Biometrics. Pattern Recognition Letters, Special Issue on Multimodal Biometrics 24 (2003) 2115-2125

4. Bigun, E.S., Bigun, J., Duc, B., Fischer, S.: Expert Conciliation for Multimodal Person Authentication Systems using Bayesian Statistics. In: Proceedings of First International Conference on AVBPA, Crans-Montana, Switzerland (1997) 291-300

5. Kittler, J., Hatef, M., Duin, R.P., Matas, J.G.: On Combining Classifiers. IEEE Transactions on Pattern Analysis and Machine Intelligence 20 (1998) 226-239

6. Lam, L., Suen, C.Y.: Optimal Combination of Pattern Classifiers. Pattern Recognition Letters 16 (1995) 945-954

7. Wang, Y., Tan, T., Jain, A.K.: Combining Face and Iris Biometrics for Identity Verification. In: Proceedings of Fourth International Conference on AVBPA, Guildford, U.K. (2003) 805-813

8. Toh, K.A., Jiang, X., Yau, W.Y.: Exploiting Global and Local Decisions for Multimodal Biometrics Verification. IEEE Transactions on Signal Processing 52 (2004) 3059-3072

9. Snelick, R., Uludag, U., Mink, A., Indovina, M., Jain, A.K.: Large Scale Evaluation of Multimodal Biometric Authentication Using State-of-the-Art Systems. IEEE Transactions on Pattern Analysis and Machine Intelligence 27 (2005) 450-455

10. Jain, A.K., Nandakumar, K., Ross, A.: Score Normalization in Multimodal Biometric Systems. To appear in Pattern Recognition (2005)

11. Griffin, P.: Optimal Biometric Fusion for Identity Verification. Identix Corporate Research Center Preprint RDNJ-03-0064 (2004)

12. Prabhakar, S., Jain, A.K.: Decision-level Fusion in Fingerprint Verification. Pattern Recognition 35 (2002) 861-874

13. Wand, M.P., Jones, M.C.: Kernel Smoothing. Chapman \& Hall, CRC Press (1995)

14. Nelsen, R.B.: An Introduction to Copulas. Springer (1999)

15. Cherubini, U., Luciano, E., Vecchiato, W.: Copula Methods in Finance. Wiley (2004)

16. Turk, M., Pentland, A.: Eigenfaces for Recognition. Journal of Cognitive Neuroscience 3 (1991) 71-86

17. Jain, A.K., Hong, L., Bolle, R.: On-line Fingerprint Verification. IEEE Transactions on Pattern Analysis and Machine Intelligence 19 (1997) 302-314

18. Jain, A.K., Ross, A., Pankanti, S.: A Prototype Hand geometry-based Verification System. In: Proceedings of Second International Conference on AVBPA, Washington D.C., USA (1999) 166-171

19. National Institute of Standards and Technology: NIST Biometric Scores Set. Available at http://http://www.itl.nist.gov/iad/894.03/biometricscores (2004) 